\title{
BMJ Open Patient and physician attitudes regarding risk and benefit in streamlined development programmes for antibacterial drugs: a qualitative analysis
}

Thomas L Holland, ${ }^{1,2}$ Stephen Mikita, ${ }^{3}$ Diane Bloom, ${ }^{4}$ Jamie Roberts, ${ }^{5}$ Jonathan McCall, ${ }^{5}$ Deborah Collyar, ${ }^{3}$ Jonas Santiago, ${ }^{6}$ Rosemary Tiernan, ${ }^{6}$ Joseph Toerner ${ }^{6}$

To cite: Holland TL, Mikita S, Bloom D, et al. Patient and physician attitudes regarding risk and benefit in streamlined development programmes for antibacterial drugs: a qualitative analysis. BMJ Open 2016;6:e013561. doi:10.1136/bmjopen-2016013561

- Prepublication history and additional material is available. To view please visit the journal (http://dx.doi.org/ 10.1136/bmjopen-2016013561)

Received 21 July 2016 Revised 11 October 2016 Accepted 21 October 2016

CrossMark

For numbered affiliations see end of article.

Correspondence to Dr Thomas L Holland; thomas.holland@duke.edu

\section{ABSTRACT}

Objectives: To explore patient, caregiver and physician perceptions and attitudes regarding the balance of benefit and risk in using antibacterial drugs developed through streamlined development processes. Design: Semistructured focus groups and in-depth interviews were conducted to elicit perceptions and attitudes about the use of antibacterial drugs to treat multidrug-resistant infections. Participants were given background information about antibiotic resistance, streamlined drug development programmes and FDA drug approval processes. Audio recordings of focus groups/interviews were reviewed and quotes excerpted and categorised to identify key themes.

Participants: Two primary stakeholder groups were engaged: one comprising caregivers, healthy persons and patients who had recovered from or were at risk of resistant infection ( $\mathrm{N}=67 ; 11$ focus groups); and one comprising physicians who treat resistant infections $(\mathrm{N}=23)$.

Results: Responses from focus groups/interviews indicated widespread awareness among patients/ caregivers and physicians of the seriousness of the problem of antibacterial resistance. Both groups were willing to accept a degree of uncertainty regarding the balance of risk and benefit in a new therapy where a serious unmet need exists, but also expressed a desire for rigorous monitoring and rapid, transparent reporting of safety/effectiveness data. Both groups wanted to ensure that $>1$ physician had input on whether to treat patients with antibiotics developed through a streamlined process. Some patients/ caregivers unfamiliar with exigencies of critical care suggested a relatively large multidisciplinary team, while physicians believed individual expert consultations would be preferable. Both groups agreed that careful oversight and stewardship of antibacterial drugs are needed to ensure patient safety, preserve efficacy and prevent abuse.

Conclusions: Groups comprising patients/caregivers and physicians were aware of serious issues posed by resistant infections and the lack of effective

\section{Strengths and limitations of this study}

- The qualitative approaches used in this study allowed for in-depth exploration of attitudes and perceptions among different groups of key stakeholders directly affected by streamlined development programmes for antibacterial drugs.

- Since interview participants were asked to reflect on past experiences, their reported impressions may be subject to recall bias.

- Study participants comprised a non-random, purposive sample, and it is possible that those who agreed to take part in this research may be different from those who did not, which may affect the generalisability of the findings.

- Detailed demographic and baseline data for participants were not gathered.

antibacterial drug therapies and shared a consensus that streamlined development programmes represent a necessary response to the resistance crisis, but one that requires enhanced safeguards and risk communication.

\section{BACKGROUND}

Infections due to resistant and multidrugresistant (MDR) bacteria are proliferating worldwide. The WHO reports 'very high' rates of resistance globally for ubiquitous pathogenic bacteria such as Escherichia coli, Klebsiella pneumoniae and Staphylococcus aureus, ${ }^{1}$ and the Centers for Disease Control and Prevention (CDC) estimates that in the USA alone $\sim 2$ million people are infected with resistant strains of bacteria each year, 23000 of whom die as a consequence. ${ }^{2}$ The danger posed by resistant bacterial infections 
is exacerbated by a paucity of newer effective antibacterial drugs capable of replacing older and increasingly ineffective treatments ${ }^{3-8}$ and further complicated by various challenges affecting current drug development processes. $^{9-13}$

To address this serious public health threat, the US Food and Drug Administration (FDA) has issued a draft Guidance for Industry for researchers working to develop new antibacterial therapies for these unmet medical needs. ${ }^{14}$ This guidance discusses approaches to streamlining drug development in order to make promising treatments available to patients with serious infections whose conditions meet the FDA's definition of unmet need, which specifies (1) "...a condition whose treatment or diagnosis is not addressed adequately by available therapy" and (2) "...an immediate need for a defined population (ie, to treat a serious condition with no or limited treatment) or a longer term need for society (eg, to address the development of resistance to antibacterial drugs)." ${ }^{14}$ Although this process could facilitate access to new antibacterial drugs for the people who need them most, the safety and efficacy data collected through such streamlined approaches will invariably be less comprehensive than data for drugs evaluated through traditional drug development pathways.

To date, the attitudes of patients and providers towards the use of antibacterial drugs developed using streamlined approaches have not been well characterised in the literature. However, because antibacterial drugs developed in this manner will almost always be associated with a greater degree of uncertainty regarding their safety and efficacy, a better understanding of the knowledge and opinions of patients, their caregivers and physicians regarding the use of these therapies can help to better inform risk communication and stewardship efforts and may have important public health implications. For these reasons, the Clinical Trials Transformation Initiative (CTTI), ${ }^{15}$ a public-private partnership whose members include representatives from the FDA, the Centers for Medicare and Medicaid Services (CMS) and academic, industry, and patientadvocacy groups, conducted a series of focus groups and in-depth interviews designed to explore patient, caregiver and physician perceptions and attitudes regarding the trade-offs between benefit and risk when using antibacterial drug therapies developed through nontraditional or 'streamlined' approaches.

\section{METHODS \\ Participants}

This research project explored attitudes and perceptions of patients and healthcare providers towards the use of antibacterial drugs developed for treatment of resistant organisms in a streamlined manner. Qualitative methods were chosen for this research because the open-ended nature of this methodology allows for a full exploration of the issues under investigation. A series of focus groups and in-depth telephone interviews, each lasting 1-2 hours, were conducted with two groups: (1) 'patients/caregivers' and (2) 'physicians' (detailed descriptions provided below).

Participants in the patient/caregiver group were recruited through flyers placed in clinics and other locations around Duke University Medical Center and other parts of Duke campus. The flyers explained the study and asked those who met study criteria to call if they were interested in participating in a focus group. Classified online advertisements were also placed on Craigslist in New York; Washington, DC; Philadelphia, Pennsylvania; and San Francisco, California to recruit participants for the patient/caregiver groups. Those who called were screened for eligibility (table 1) and scheduled into the appropriate group.

Participants in the physician group were contacted from a list of experts in relevant fields developed in consultation with the study research committee. These lists were developed by requesting members of our interdisciplinary project team, including those from FDA and

Table 1 Eligibility criteria for patient/caregiver group

\begin{tabular}{ll}
\hline Group & Criteria \\
\hline All participants & $\begin{array}{l}\text { Provide oral informed consent } \\
\text { Speak and understand English }\end{array}$ \\
$\begin{array}{l}\text { Group 1: Healthy } \\
\text { patients }\end{array}$ & $\begin{array}{l}\text { Have not had a resistant or hospital-acquired infection } \\
\text { Do not have a chronic illness that places them at risk for frequent hospitalisations and resulting } \\
\text { hospital-acquired infection }\end{array}$ \\
$\begin{array}{ll}\text { Group 2: Recovered } \\
\text { patients }\end{array}$ & $\begin{array}{l}\text { Have recovered from a resistant or hospital-acquired infection } \\
\text { Do not have a chronic illness that places them at risk for frequent hospitalisations and resulting } \\
\text { hospital-acquired infection } \\
\text { Group 3: At-risk patients }\end{array}$ \\
$\begin{array}{l}\text { Have a chronic illness that puts them at risk for frequent hospitalisations and resulting } \\
\text { hospital-acquired infections } \\
\text { May or may not have had a resistant or hospital-acquired infection } \\
\text { *'Caregiver' denotes family/friend/other caregiver, as distinct from a healthcare professional (or 'provider'). }\end{array}$
\end{tabular}


the CTTI Steering Committee, to provide us with names of physician experts in the disciplines of infectious disease (ID), critical care, intensive care, pulmonary care and others (including hospitalists). These persons were then approached by the project manager and invited to participate in this study based on the recommendation of a project team member or CTTI Steering Committee member. We also used the services of Schlesinger Associates, a qualitative research recruiting firm specialising in physician recruitment, to identify and schedule some of the specialists.

Discussion guides and informational handouts for both groups were developed iteratively in consultation with the study research committee, which included representatives from the FDA, expert physicians and clinical investigators from the Duke Clinical Research Institute. All focus groups and in-depth interviews were conducted by an experienced independent social scientist and a professional moderator (DB). The researcher was a trained professional for whom this study represented her first qualitative study in this particular topic area; she was neutral regarding the topic area. Participants in the patient/caregiver focus groups were not given information about the study sponsor in an attempt to avoid introducing bias. ${ }^{16} 17$

The interviewer moderated three in-person focus groups at a conference room at the Duke Clinical Research Institute in Durham, North Carolina. In-person group sessions lasted $\sim 1.5-2$ hours. An interview guide specific to the patient/caregiver group was used by the moderator to inform the course of the sessions but not necessarily followed point-by-point, based on the moderator's discretion. Remaining focus groups were conducted by telephone to obtain broader geographical distribution.

For the provider group, one in-person interview with a single participant was conducted at the American Thoracic Society International Conference in Denver, Colorado (May 15-20, 2015). This interview was used as a pilot study, and the interview guide specifically designed for providers was revised according to responses received in this session. The remaining provider interviews were conducted by telephone on a one-on-one basis.

All focus groups and interviews were audio recorded. An open-ended and exploratory approach was used in which the interviewer reviewed all audio recordings from focus groups and in-depth interviews, making detailed notes that included verbatim quotations, in preparation for a systematic qualitative analysis performed in accordance with standard methods for qualitative research. ${ }^{16-20}$ All quotations were assigned to a content category from one of the constructs in the discussion guide. The interviewer then reviewed quotes in each category, noting which points of view prevailed and which were less common, in order to identify the strongest themes that emerged. Formal coding was not used in this study, nor was any specialised software used to categorise responses. Data saturation ${ }^{21}$ was reached with provider and patient/caregiver groups.

\section{Group 1: patients and caregivers}

\section{Eligibility criteria}

Using a semistructured topic guide, DB conducted 11 focus groups (total $\mathrm{N}=62$ ). These focus groups comprised four major categories of participants: (1) healthy patients, (2) recovered patients, (3) at-risk patients and (4) caregivers of groups 2 and 3 (table 1).

Baseline, demographic and other data about health status (other than that noted above) were not gathered for study participants. All participants understood and spoke English and provided oral informed consent, as described below.

Prior to taking part in a focus group, all participants were mailed a scenario for discussion and 'fact-sheet' summaries describing antibacterial drug resistance (NB: for patient/caregiver focus groups, the terms 'antibiotic' and 'antibiotic resistance' were used), the FDA's current drug review and approval process and a summary of the draft FDA guidance concerning streamlining development for antibacterial drugs/biologics that address unmet medical need (see online supplementary appendix 1 ).

\section{Group 2: healthcare providers}

A series of in-depth telephone interviews and a single in-person interview were conducted by DB with physicians (total $\mathrm{N}=23$ ) who represented a mix of academic $(n=11)$ and community-based $(n=12)$ practices from multiple specialties that are typically involved in identifying, diagnosing and treating resistant and MDR infections, including hospitalists, intensivists, pulmonology/ critical care and IDs. All were provided with scenarios and an informational handout (see online supplementary appendix 2) about streamlined development as triggers for discussion. Physicians were asked about their experiences treating patients with MDR infections, and their perspectives regarding use of FDA-approved antibacterial drugs developed using a streamlined approach were explored.

\section{Eligibility criteria}

Participants in the healthcare provider group were selected based on criteria applied by the CTTI project committee, as described above.

\section{Study approval and informed consent}

The study protocols for the patient/caregiver and provider groups were submitted to the Duke University Health System institutional review board (IRB). The Duke IRB deemed the provider focus group/interview study to be exempt from review. Patient/caregiver focus groups/interviews were conducted under an alteration of informed consent and waiver of authorisation for use or disclosure of information protected by the Health Insurance Portability and Accountability Act (HIPAA). All prospective participants received a copy of the study 
consent form via postal mail and were offered the opportunity to ask questions of study personnel. Participants provided oral consent prior to starting study activities. Study flyers used to recruit participants were reviewed and approved by the Duke IRB as well.

\section{RESULTS}

\section{Patient/caregiver focus group responses}

A detailed listing of themes that emerged from focus groups with patients and caregivers is presented in box 1 .
Patient perceptions: attitudes about antibacterial drugs

The majority of participants said they are wary of taking any prescription medications (including antibiotics) because of the risks and side effects associated with almost all of them. Most prefer to 'wait it out' make lifestyle changes or take natural remedies, which they perceive as safer than prescription drugs. However, 'wonder drugs' and 'miracle drugs' were words some recalled hearing to describe antibacterial drugs, because they cure infections that used to be deadly. When probed, most did view antibacterial drugs as safer

Box 1 Major themes and detailed responses from the patient/caregiver group

Mindset regarding prescription medications

- Wary of prescription drug use*

Concerned about potential risks and side effects*

- See antibiotic drugs in more positive light*

- Theoretically prefer natural/homeopathic remedies or lifestyle changes, but also value life-saving medications†

- Underlying health conditions make them vulnerable to infection†

Perceptions of existing and new antibacterial drugs

- Most viewed antibiotics as safer than many other prescription drugs

- Most do not believe that 'newer is better'

- Most adopt 'wait and see' attitude towards adopting new medication

Perceptions of antibacterial resistance

- Almost all familiar with general concept of antibiotic resistance

- Many mistakenly thought overuse leads to resistance in individuals

- All were surprised by magnitude of problem

- All were surprised that 'superbugs' can be transmitted from person to person

- All believe new antibiotics are urgently needed

Perceptions of FDA drug review process

- Almost all surprised at potential length of process

- Confidence in process eroded by recalls and DTC advertisement listing side effects

Perceptions of streamlined approaches

- All reacted positively to description of streamlined development

- Saw advantage in getting new antibacterial drugs to critically ill patients with few/no additional options

- Concerned about abuse/overuse by industry

Use in situations of critical need

- Most would not want to rely on judgement of single doctor

- Suggested an interdisciplinary 'A-Team' to oversee use decision

- Most would find decision-making on behalf of loved one more difficult

- Would like information in advance to let doctors/family know wishes $†$

- Almost all believed that if critically ill, they would not be able to make decisions

Information desired about drugs approved using streamlined approaches

- In how many people/what populations has drug been tested?

-What are the side effects, including most common and most serious?

- What drug interactions have been observed?

- How effective is drug, and what else is known about the drug?

- How do they know they actually have a resistant infection?

- What are the odds of survival without the medication?

-What would likely happen if they do not take the medication?

- Who decides to offer the new antibacterial drug, and what are alternatives?

*Included healthy patients, recovered patients and family/caregivers.

tIncluded patients at risk for developing a resistant infection.

Additional details available in online supplementary appendix 3.

DTC, direct-to-consumer; FDA, US Food and Drug Administration. 
than many other kinds of prescription drugs, in part because of the short-term nature of most antibacterial drug regimens. Additionally, most had positive experiences with antibacterial drugs as their infections resolved with few or no serious side effects (see online supplementary box 1$)$.

\section{Patient perceptions: existing versus new antibacterial drugs}

Regarding new medications, including antibacterial drugs, most subscribed to the view that 'newer isn't always better', with most indicating that 'new' connotes 'not fully tested yet'. Some cited examples of promising new drugs that were touted as effective and safe but later were found to cause side effects serious enough to warrant withdrawing the drug from the market. As a result of such reversals, most indicated a wait-and-see attitude towards taking brand-new medications, preferring to take older medications that are 'tried and true'. However, all agreed that if they were very ill and out of options, they would be grateful to have new, effective therapies.

\section{Patient perceptions: antibacterial drug resistance}

Almost all participants were familiar with the concept of antibacterial resistance, saying that when antibacterial drugs are overused, they can become ineffective in treating infections they once cured. Many were concerned about overuse of antibacterial drugs in humans and livestock, identifying such overuse as a major contributor to resistance. Although participants knew that resistant infections could be life-threatening, all were surprised at the magnitude of the problem.

Most participants appeared to have an incorrect understanding of the ways in which resistance develops, including the assumption that persons who take too much of a particular antibacterial drug will build up an individual tolerance to it, rendering that drug ineffective for them personally (see online supplementary box 2). Even after explanations of antibacterial drug resistance, most did not appear to understand the underlying mechanisms; namely, that bacteria develop resistance to antibiotics, as opposed to individuals becoming resistant or developing tolerance to the effects of antibacterials. They also expressed surprise that resistant pathogens could be spread from person to person. All agreed that new antibacterial drugs are needed urgently to combat the growing problem of antibacterial drug resistance.

\section{Patient perceptions: drug development process}

Although participants typically shared the impression that current drug development processes are involved and lengthy, they expressed surprise that it could take as long as 15 years to develop and submit a new drug application to the FDA for review and that historically many of the drugs studied ultimately did not complete the drug development process. Participants noted, however, that the length of the development and approval processes did not increase their confidence in the new drugs, citing instances of drugs withdrawn from market due to serious, life-altering side effects and lengthy recitations of side effects in direct-to-consumer advertising (see online supplementary box 3 ).

Patient perceptions: FDA streamlined approaches for antibacterial drug development

Participants were given a handout (see online supplementary appendix 1) about a proposed development process for new antibacterial drugs that represented a streamlined approach. All applauded the concept because the problem of antibacterial resistance is dire and such a process could help bring new antibacterial drugs more quickly to people who need them. They cited access to potentially life-saving treatments for critically ill patients with limited options as a major benefit of a streamlined process, while the major disadvantage noted was limited data about potentially serious side effects, especially when prescribed for fragile patients with multiple comorbidities. Some expressed concern that the process to streamline development could be abused by profit-driven pharmaceutical companies seeking to fast-track other medications as well, and they wanted safeguards to ensure that streamlining does not become 'the new normal' in drug development and approval (see online supplementary box 4 ).

\section{Patient perceptions: use in unmet need contexts}

The groups of patients and caregivers discussed how they would feel about taking a new antibacterial drug developed through a streamlined approach if they were critically ill with a resistant infection. Almost all said that they would definitely take the new antibacterial drug. Most said that they likely would be too ill to do research about the drug and would say, 'Bring me the pill' and not 'Bring me the iPad!' (see online supplementary box 5).

Most said, however, that they would not want to rely on the judgement of a single physician about whether to use an antibacterial therapy developed using a streamlined approach. Some expressed a preference for a multidisciplinary team-their personal physician, a patient advocate, hospitalist, immunologist or other expert(s) - to guide the use of such products. Their primary concern was that more than one physician be involved in the decision, however, and they were not wedded to the idea of a large team. Some participants at high risk of serious infection indicated that they would prefer to make their feelings about this kind of treatment known to their doctors before their medical condition became critical in a manner similar to that used in an advanced directive, because they likely would not be able to make informed, deliberate decisions about such therapies in acute settings. However, most of the at-risk patients indicated that if faced with a lifethreatening situation, they would take an antibacterial drug developed using a streamlined approach (see online supplementary box 6 ). 
Patient perceptions: information desired about antibacterial drugs developed using a streamlined approach

Focus group participants said that they would like the following kinds of information when considering whether to take an approved antibacterial drug developed using a streamlined approach: (1) how many people has the drug been tested on; (2) what are the most serious and the most common side effects; (3) how effective is the drug; (4) how accurate is the diagnosis of antibacterial drug-resistant infection; (5) what are their odds of survival without the new antibacterial drug and what are the likely consequences if they are not treated with the new antibacterial drug; (6) who decides whether patients will be offered the new therapy and (7) what, if any, treatment alternatives exist.

\section{Physician perspectives}

The themes that emerged from in-depth interviews with physicians who treat resistant infections in the course of their practices are detailed in box 2 and online supplementary appendix 4 .
Physician perceptions: challenges in treating patients with complicated infections

Physicians identified two overarching challenges when treating patients with serious, resistant or complicated infections: (1) choosing appropriate therapeutics before definitive identification of pathogens through culture is available; and (2) treating very ill patients who require close monitoring and whose response to antibacterial drug therapies with significant toxicities may be unpredictable.

\section{Physician perceptions: attitudes towards a streamlined development approach}

Respondents agreed that a crisis exists with regard to MDR infections and that streamlining development is an appropriate approach towards resolving this problem. Almost all believed the benefits of antibacterial drugs approved after undergoing this type of development outweighed the risks for critically ill patients with limited options. Some also said that the benefits given to the pharmaceutical companies for developing new antibacterial drugs are appropriate in order to create incentives

Box 2 Major themes and detailed responses from the physician group

Greatest challenges in treating patients with complicated infections

- Choosing appropriate treatment before definitive culture available

- Treating very ill/fragile patients requiring close monitoring where tolerance and toxicity are difficult to predict

Perceptions of streamlined approaches

- All believed there is a crisis in antibacterial-resistant infection treatment and streamlined development approaches are an appropriate measure

Use of approved antibacterial drugs developed using a streamlined approach

- None believed these products should be frontline therapies; all would wait for definitive culture results before using

- Would use only for patient with true unmet needs who have exhausted options

Concerns regarding antibacterial drugs developed using a streamlined approach

- Most were not concerned about using such drugs in critically ill patients with unmet needs

- Some expressed concern about lack of efficacy and safety data (esp. renal toxicity)

- Before using for patients with HABP/NABP, would want to know sensitivity to pathogens and whether it had sufficient lung penetration

Confidence regarding use of a drug developed using streamlined approaches

- Confidence would be boosted by FDA approval and vetting by hospital P\&T Committee

Restrictions on use

- Few existing restrictions on ID/critical care physicians' prescribing authority

- Should be mandatory consults with ID physicians on hospital stewardship committee/experts in multidrug-resistant infections

- Some wished to ensure that ID consults would not interfere with timely use in critical situations

Ongoing need for information

- Some expressed desire for continuous updates on efficacy/safety

- Some suggested data from use in clinical settings be continuously submitted to trusted neutral third party to inform physicians and create guidelines

Bedside consult

- All believe multidisciplinary 'A-Team' was neither necessary or desirable and that a mandatory ID consult would suffice

Patient advance directive for the use of antibacterial drugs developed using a streamlined approach

- None thought 'advance directive' regarding use of such a drug would be practicable or desirable

Additional details available in online supplementary appendix 4.

HABP, hospital-acquired bacterial pneumonia; ID, infectious diseases; P\&T, pharmacy and therapeutic; VABP, ventilator-associated bacterial pneumonia. 
for the development of new medications that are unlikely to be 'blockbuster' drugs.

Although a few participants expressed concerns about the safety of such therapies and the medicolegal risk associated with their use, most said that they would feel comfortable using a drug developed through this approach, even with relatively smaller amounts of supporting data, because most drugs currently used for treating resistant infections are 'suboptimal', with known potentially serious toxicities, most notably nephrotoxicity. Most indicated that they would be more confident using these drugs because they knew they had passed the FDA approval processes as well as those applied by their own institutions' Pharmacy and Therapeutics (P\&T) Committees. Familiarity with one or more of the drug's components would further increase their comfort levels.

There was a consensus that antibacterial drugs developed through streamlined processes should be used only for patients with limited options and only after the pathogens were identified through cultures. The majority said they would likely not use these antibacterial drugs as 'first-line' therapies even after the pathogens were identified, in order to preserve the new drug for patients with true unmet medical need. Most indicated that they were not concerned about using new antibacterial drugs developed using a streamlined process, although a minority expressed concerns about lack of efficacy and limited safety data. Respondents also said they would need specific data on a particular drug's pulmonary penetration (in order to better form an impression of the drug's likely efficacy) before using it in patients with pneumonia.

\section{Physician perceptions: restrictions on use of antibacterial drugs developed via a streamlined approach}

Almost all respondents said that the use of these therapeutics should include mandatory consultations with ID specialists, preferably ones who serve on the hospital antibiotic stewardship committee, or ones who are experts in treating resistant infections. A few suggested creating a special certification in multidrug resistance within the ID specialty in order to designate experts in this arena. Some expressed concerns that such consultations could interfere with timely administration of potentially life-saving medication, suggesting that, in extenuating circumstances, patients be given the new medication with the understanding that there would be an expert review within 24-48 hours.

\section{Physician perceptions: need for continuous data collection and reporting}

Respondents indicated that efficacy and safety data from the use of antibacterial drugs developed using a streamlined approach in practice settings be continuously collected and submitted to a neutral, trusted third party that would inform physicians and establish treatment guidelines for new antibacterial drugs.

\section{Physicians: communication with patients and family}

The physicians interviewed in this study said that patients with resistant infections are typically too ill to engage in a discussion about their condition, and family members generally lack the detailed medical knowledge needed to play a role in making treatment choices that even specialists find complicated. The patient's family can provide physicians with information about patients' allergies and infection history. Physicians in turn can tell the family what type of infection they think the patient has and which antibacterial drug they feel is the best match for the infection, as well as describing potentially serious side effects and what can be performed to reduce risks. Physicians who had experience with approved antibacterial drugs developed using a streamlined process (eg, the ceftazidime/avibactam combination therapy used to treat MDR Gram-negative bacterial infections) said they do not tell families that the treatment drug was approved based on data from a streamlined development programme, noting that the practice of using other therapies with limited data to support their use is commonplace in inpatient settings.

\section{Physician perceptions: bedside consultation and advanced directives}

None of the physicians believed that a multidisciplinary team would be useful in making determinations about whether to use an antibacterial drug developed through a streamlined approach. All thought that convening such a team could delay critical treatments and that a mandatory consultation by the ID specialist would serve the same purpose of assuring patients that the decision was being made by more than one physician with relevant expertise. In addition, none thought a patient 'advance directive' concerning the use of such therapies would be feasible or useful because patients' general practitioners, although they are kept informed, are typically not involved in daily decision-making once a patient is admitted to the hospital.

\section{DISCUSSION}

In the focus group discussions and interviews with the patient/caregiver and physician groups, several key areas of convergent opinions were noted (table 2).

First, there was widespread awareness of the seriousness of the problem of antibacterial resistance among patients/caregiver and physician groups. In addition, participants' overall reactions to the use of new antibacterial drugs developed using streamlined approaches suggest that patients/caregivers and physicians are willing to accept a degree of uncertainty regarding the balance of risk and benefit in a new therapy for a condition where a serious unmet medical need exists, particularly if such therapies are accompanied by rigorous oversight, continuous monitoring and rapid and transparent reporting of safety and effectiveness data. Further, for both groups the degree of uncertainty 
Table 2 Key areas of convergence and disagreement between patient/caregiver and physician groups

Convergence
Widespread awareness of problem of antibiotic resistance
and agreement that it represents a serious problem

Streamlined development represents a reasonable response to crisis

Decisions to use antibiotics approved under a streamlined process should be made by more than one physician with relevant experience

Willingness to accept degree of uncertainty in risk/benefit balance, increasing with severity of illness

Decision-making regarding use of therapies developed with a streamlined approach requires more than one person

Safeguards needed to prevent abuse of streamlined

development

\section{Disagreement}

Some patients/caregivers expressed preference for relatively large multidisciplinary decision-making team, including primary care provider and ID specialist; physicians favoured a mandatory expert consultation to assure patients of appropriate care

Understanding of nature and mechanisms of antibiotic resistance (many patients had an erroneous understanding that could potentially affect adherence to treatment and patient well-being)

ID, infectious disease.

considered tolerable increases with the severity of the circumstances and the lack of alternative therapies. Finally, responses from both groups were premised with a broad agreement concerning the need for careful stewardship of antibacterial drug therapies, both to protect patients from the effects of therapies whose balance of benefit and risk might not be fully characterised and to preserve the effectiveness of the therapy.

Both groups also showed broad agreement regarding the desirability and usefulness of streamlining development for novel antibacterial therapies with potential for addressing currently unmet needs. Both groups also believed that such therapies should be used only in patients with limited therapeutic options and that safeguards and oversight are needed to prevent streamlining from becoming 'the new normal' for antibacterial drug development (and for other types of drugs). Further, physicians and patients/caregivers agreed on the need for continuous monitoring and reporting of data, preferably in real-time, regarding the safety and efficacy of approved therapies developed using a streamlined approach. Of note, these views are also reflected in the US Code of Federal Regulations. ${ }^{22}$

In addition to these areas of convergence, there were also indications of divergent opinions between the patient/caregiver and physician groups in issues relating to the decision-making process about whether to use therapies developed using streamlined approaches. Although both groups agreed that more than one person should be involved and that experts should be included in the consultation, some patients/caregivers expressed a preference for a relatively large multidisciplinary decision-making team, including their primary care provider and an ID specialist, while all of the physicians viewed such a measure as unrealistic and likely to delay treatment, instead favouring a mandatory expert consultation which would assure patients of appropriate care without engendering potentially dangerous delay.

Similarly, some at-risk patients and their caregivers favoured the idea of establishing an 'advance directive' with their personal physicians concerning their wishes about the use of such therapies should the need arise. Physicians more familiar with the medical system considered such a measure unhelpful, because general practitioners are typically not involved in treatment decisions after their patients are admitted. Also, physicians noted that many other procedures and treatments used for hospitalised patients are often not supported by definitive evidence or comprehensive data.

These relatively limited divergences of opinion between physicians and patients/caregivers may reflect the fact that patients would not necessarily be familiar with the details of specific hospital procedures and protocols employed in life-threatening situations where timely action is critically important to patient well-being. Similarly, patient/caregiver groups also would not be expected to know about the rigorous multidisciplinary vetting provided by hospital P\&T committees for new therapeutics-information that might alleviate some of their concerns.

We observed that the majority of patients and caregivers seemed to share misperceptions about the nature of antibacterial drug resistance and its underlying mechanisms that could have serious implications for patients at risk for recurrent hospitalisation and infections, as such misperceptions could potentially lead to patients deferring life-saving antibacterial drugs in hopes of preserving future options. Of note, although our study sample was relatively small and limited to the USA, a recent large-scale $(\mathrm{N}=9772)$, multinational survey by the WHO demonstrated similarly widespread 
misunderstandings about antibiotic resistance, with $76 \%$ of those surveyed indicating a belief that resistance arises when their bodies become individually habituated to antibiotic drugs. ${ }^{23}$ It is also important to recognise that at-risk patients and caregivers all indicated that they would take drugs developed through a streamlined approach if they were critically ill with a resistant infection and had few or no other treatment options.

\section{Limitations}

We note a number of limitations to our study. First, although qualitative research techniques can provide useful and detailed insights into a target audience's perceptions and beliefs, such data by definition are not as clear-cut as objective data and conclusions drawn from them may not be generalisable to larger audiences. Rather, these findings are intended to provide a sense of what particular samples of patients and physicians know and feel regarding MDR infections and about the use of approved antibacterial drugs which were developed using a streamlined approach. In addition, in interviews with recovered and at-risk patients and caregivers, respondents are prompted to look back on their past experiences with resistant infections. Such retrospective techniques may not accurately elicit their thinking in the midst of these experiences, and their reported impressions may be subject to bias.

Although the duration of focus group sessions and interviews could potentially present challenges in terms maintaining engagement and avoiding participant fatigue, the length of sessions in this study (1.5-2 hours for in-person focus groups; $\sim 1$ hour for telephone groups) was typical of similar studies. The presence of an experienced moderator also helped to maintain engagement in in-person and telephone sessions, as did varied activities and discussion of handouts.

Streamlining development could potentially bring new antibacterial drugs to market sooner if sponsors more efficiently design and conduct clinical trials to generate the data needed to assess risks and benefits of investigational drugs. However, it is important to note that a streamlined drug development programme can meet FDA's statutory requirements for drug approval-showing that the drug is safe and effective for its indicated use-and FDA would then evaluate the clinical efficacy and safety data information made available with its usual level of rigour.

There are also limitations to our sampling methodology. The patients and physicians interviewed represented a non-random, purposive sample recruited as representative of the target population, and it is possible that those who agreed to take part in this research may be different from those who did not, which may have implications for the generalisability of the findings. However, this is an accepted method for recruiting respondents for qualitative research. Our study did not collect detailed baseline characteristics or demographic data, which might have provided additional useful information about study participants. In addition, the focus group effort did not fully explore bedside risk/benefit decision issues related to whether a patient had 'limited' versus 'no options'.

In conclusion, there is substantial support from patients/ caregivers and providers for streamlined development approaches as a necessary response to the current antibacterial resistance crisis. However, the use of approved antibacterial drugs developed using a streamlined approach will require increased safeguards and careful risk communication and stewardship to protect against potential harms for individual patients and for society.

\section{Author affiliations}

${ }^{1}$ Department of Medicine, Duke University School of Medicine, Durham, North Carolina, USA

${ }^{2}$ Duke Clinical Research Institute, Durham, North Carolina, USA

${ }^{3}$ Patient Advocates in Research (PAIR), Danville, California, USA

${ }^{4}$ InFocus Research, Chapel Hill, North Carolina, USA

${ }^{5}$ Duke Clinical Research Institute, Durham, North Carolina, USA

${ }^{6}$ Center for Drug Evaluation and Research, US Food and Drug Administration, Silver Spring, Maryland, USA

\section{Twitter Follow Deborah Collyar at @deborahcollyar}

Acknowledgements The authors acknowledge the efforts and contributions of the full CTTI Unmet Need in Antibacterial Drug Development Project team, including Ed Cox (FDA), Helen Donnelly (Northwestern University), Vance Fowler (Duke University) and Jeff Loutit (The Medicines Company). The Unmet Need in ABDD team designed the evidence gathering instruments and conducted the data analysis.

Disclaimer Views expressed in written materials or publications and by speakers and moderators do not necessarily reflect the official policies of the Department of Health and Human Services, nor does any mention of trade names, commercial practices or organisations imply endorsement by the US Government.

Contributors RT, JS, JT, TLH, SM and JR conceived the study. DB developed prompts and questions, conducted focus groups and interviews and coded responses. RT, JS, JT, TLH, SM, DC and JR analysed and interpreted the results. JM drafted the manuscript, and all authors contributed revisions to subsequent drafts. All authors read and approved the final manuscript.

Funding This work was made possible in part by the US Food and Drug Administration through grant R18FD005292 and cooperative agreement U19FD003800. Partial funding was also provided by pooled membership fees from the Clinical Trials Transformation Initiative's member organisations.

Competing interests Three authors (JS, RT, JT.) are employees of the US Food and Drug Administration, a funder of the Clinical Trials Transformation Initiative and the present study. The FDA is responsible for the review and approval of antibacterial drugs, including those developed using streamlined approaches.

Ethics approval Ethical approval for this study was granted by the Duke University Health System Institutional Review Board.

Provenance and peer review Not commissioned; externally peer reviewed.

Data sharing statement Unpublished data comprising summary reports with transcribed responses from individual focus groups, individual provider interview summaries, redacted pilot study transcripts, and model consent forms are available on request from the corresponding author.

Open Access This is an Open Access article distributed in accordance with the Creative Commons Attribution Non Commercial (CC BY-NC 4.0) license, which permits others to distribute, remix, adapt, build upon this work noncommercially, and license their derivative works on different terms, provided the original work is properly cited and the use is non-commercial. See: http:// creativecommons.org/licenses/by-nc/4.0/ 


\section{REFERENCES}

1. World Health Organization. Antimicrobial resistance: global report on surveillance. 2014. http://apps.who.int/iris/bitstream/10665/112647/1/ WHO_HSE_PED_AIP_2014.2_eng.pdf?ua=1 (accessed 12 Nov 2015).

2. Centers for Disease Control and Prevention. Antibiotic resistance threats in the United States, 2013. http://www.cdc.gov/ drugresistance/threat-report-2013/ (accessed 12 Nov 2015).

3. Spellberg B, Powers JH, Brass EP, et al. Trends in antimicrobial drug development: implications for the future. Clin Infect Dis 2004;38:1279-86.

4. Norrby SR, Nord CE, Finch R. European Society of Clinical Microbiology and Infectious Diseases. Lack of development of new antimicrobial drugs: a potential serious threat to public health. Lancet Infect Dis 2005;5:115-9.

5. Finch R, Hunter PA. Antibiotic resistance-action to promote new technologies: report of an EU Intergovernmental Conference held in Birmingham, UK, 12-13 December 2005. J Antimicrob Chemother 2006;58(Suppl 1):i3-i22.

6. Projan SJ, Bradford PA. Late stage antibacterial drugs in the clinical pipeline. Curr Opin Microbiol 2007;10:441-6.

7. Boucher HW, Talbot GH, Bradley JS, et al. Bad bugs, no drugs: no ESKAPE! An update from the Infectious Diseases Society of America. Clin Infect Dis 2009;48:1-12.

8. Nambiar S, Laessig K, Toerner J, et al. Antibacterial drug development: challenges, recent developments, and future considerations. Clin Pharmacol Ther 2014;96:147-9.

9. Bettiol $\mathrm{E}$, Wetherington JD, Schmitt $\mathrm{N}$, et al. COMBACTE Consortium. Challenges and solutions for clinical development of new antibacterial agents: results of a survey among pharmaceutical industry professionals. Antimicrob Agents Chemother 2015;59:3695-9.

10. Williams KJ, Bax RP. Challenges in developing new antibacterial drugs. Curr Opin Investig Drugs 2009;10:157-63.

11. Silver LL. Challenges of antibacterial discovery. Clin Microbiol Rev 2011;24:71-109.
12. Echols RM. Understanding the regulatory hurdles for antibacterial drug development in the post-Ketek world. Ann N Y Acad Sci 2011;1241:153-61.

13. Echols RM. A long and winding road; evolution of antimicrobial drug development-crisis management. Expert Rev Anti Infect Ther 2012;10:1311-9.

14. US Food and Drug Administration. Draft guidance for industry: antibacterial therapies for patients with unmet medical need for the treatment of serious bacterial diseases. July 2013. http://www.fda. gov/downloads/drugs/guidancecomplianceregulatoryinformation/ guidances/ucm359184.pdf (accessed 12 Nov 2015).

15. Clinical Trials Transformation Initiative. Who we are. http://www. ctti-clinicaltrials.org/who-we-are (accessed 12 Nov 2015).

16. Corbin J, Strauss A. Basics of qualitative research: techniques and procedures for developing grounded theory. 3rd edn. Thousand Oaks, CA: Sage Publications, 2008.

17. Patton MQ. Qualitative research and evaluation methods. London: Sage Publications, 2002

18. Goldman AE, McDonald SS. The group depth interview: principles and practice. Englewood Cliffs, NJ: Prentice-Hall, 1987:7-23.

19. McCracken G. The Long Interview. Newbury Park, CA: Sage Publications, 2008.

20. Green J, Thorogood N. Qualitative methods for health research. 2nd edn. Thousand Oaks, CA: Sage Publications, 2009.

21. Guest G, Bunce A, Johnson L. How many interviews are enough? An experiment with data saturation and variability. Field Methods 2006;18:59-82.

22. Code of Federal Regulations. 21 CFR 312.80. Subpart E. Drugs intended to treat life-threatening and severely-debilitating illnessespurpose. https://www.accessdata.fda.gov/scripts/cdrh/cfdocs/cfcfr/ CFRSearch.cfm?CFRPart=312\&showFR=1\&subpartNode=21:5.0.1. 1.3.5 (accessed 11 Apr 2016).

23. World Health Organization. Antibiotic resistance: multi-country public awareness survey. Executive summary. http://apps.who.int/iris/ bitstream/10665/194460/1/9789241509817_eng.pdf?ua=1/ (accessed 8 Jan 2015) 